\title{
Influence of the quench rate and the pressure on the glass transition temperature in selenium
}

\author{
D. Caprion ${ }^{\text {a) }}$ and H. R. Schober \\ Forschungszentrum Jülich, IFF, D-52425 Jülich, Germany
}

(Received 20 November 2001; accepted 20 May 2002)

\begin{abstract}
We report a molecular dynamics investigation of the glass transition temperature in selenium at pressures ranging from 0 to $6 \mathrm{GPa}$ as a function of the quench rate, $Q_{r}$. For moderate pressures the specific volume of the glass depends strongly on the quench rate, whereas the specific enthalpy varies only little. We find for both volume and energy a linear dependence on the quench-rate-dependent glass transition temperature. The slopes of these curves reflect the different energy scales of void formation, inter- and intrachain interactions. The extrapolated glass transition temperatures for quench rates of order K/s agree with the experimental ones within 20\%. Applying a pressure of $1 \mathrm{GPa}$ the glass transition temperature is raised by $37 \mathrm{~K}$. For the same $Q_{r}$, the transition temperature $T_{g}$ is much higher for simulations using fixed volume conditions (NVT ensemble) than for the ones using fixed pressure ( $N P T$ ensemble) when one compares results for equal pressure at $T=0$. (C) 2002 American Institute of Physics. [DOI: 10.1063/1.1492797]
\end{abstract}

\section{INTRODUCTION}

The rapid development of computer power in recent years made it possible, using molecular dynamics simulations, to study many properties of supercooled liquids and glasses over a wide range of temperatures and on long time scales, typically nanoseconds. Such studies have been done, e.g., on model systems, soft sphere glasses ${ }^{1}$ or LennardJones glasses (LJGs), ${ }^{2,3}$ and on realistic systems such as silica, ${ }^{4-6} \mathrm{NiZr},{ }^{7} \mathrm{CuZr},{ }^{8} \mathrm{GeSe}_{2},{ }^{9} \mathrm{Se},{ }^{10,11}$ and OTP. ${ }^{12}$

The computed vibrational and structural properties are usually in reasonable agreement with experimental data, despite the large difference in the quench procedures used in simulations and experiments. In particular the quench rates, $Q_{r}$, used in simulations are typically in a range from $10^{10}$ to $10^{14} \mathrm{~K} / \mathrm{s}$, depending mainly on the system. They are, therefore, several orders of magnitude larger than in most experiments. These high $Q_{r}$ results from the technical limitations of computer simulations: for instance a quench from 600 to $0 \mathrm{~K}$ on a system of 2000 atoms of selenium with $Q_{r}=10^{12} \mathrm{~K} / \mathrm{s}$ takes about $14 \mathrm{~h}$ on 8 nodes of a Cray T3E1200 , and therefore would take about two months with $Q_{r}=10^{10} \mathrm{~K} / \mathrm{s}$. Experimentally similarly high quench rates are realized when spots are heated by laser or particle irradiation.

There are very few investigations of the dependence of the glass transition on these high quench rates or on the applied pressure. The glass transition is often studied under constant volume conditions.

Vollmayr et al. ${ }^{3,13}$ have shown that the energy of a Lennard-Jones glass at $0 \mathrm{~K}$ depends on $Q_{r}$. The smaller the $Q_{r}$, the lower the final energy. They have also shown that the system achieves a better (more perfect) local rearrange-

\footnotetext{
${ }^{a)}$ Present address: Laboratoire de Physique des Polymères, CP 223, Université Libre de Bruxelles, Bd du Triomphe, B-1050 Brussels, Belgium. Electronic mail: didier.caprion@ulb.ac.be
}

ment with lower $Q_{r}$. As found in experiment, the computed values of $T_{g}$ drop with decreasing quench rates. The same behavior was observed in a simulation of silica. ${ }^{5}$

Extrapolating the temperature and quench rate dependence of the internal energy, Vogel-Fulcher temperatures $T_{0}=0.34$ (in LJ units) and $T_{0}=2525 \mathrm{~K}$ were obtained for the LJGs and silica, respectively. In the case of LJGs the comparison with experiments is difficult since LJGs are model glasses only. In silica the experimental value of $T_{g}=1446 \mathrm{~K}$. There is a discrepancy of about $75 \%$ between experiments and estimates from simulations. ${ }^{5}$ From an extrapolation of the diffusion constant it was later argued that the glass transition of the simulated $\mathrm{SiO}_{2}$ is in broad agreement with experiment. ${ }^{6}$

Contrary to this, recent simulations on amorphous selenium show a $T_{g}$ at about $300 \mathrm{~K}$ when the experimental value is at about $305 \mathrm{~K}^{14}$ One should notice that the effective $Q_{r}$ used in these simulations is about $10^{10} \mathrm{~K} / \mathrm{s}$, which is still far above the usual experimental $Q_{r}$.

The aim of this work is to look into the origin of this discrepancy and to extend the investigations of quench rate effects to Se which, with its chain structure, represents a different class of materials than $\mathrm{SiO}_{2}$ and LJGs. Other than the above-mentioned materials $\mathrm{Se}$ is known to show a strong pressure dependence of the glass transition temperature. In the following we calculate the evolution of $T_{g}$ for different pressures with $Q_{r}$ in selenium. Additionally we investigate the effects of the ensemble used in the simulation, fixed pressure or fixed volume.

First, we briefly describe the simulations. From the temperature dependence of the specific volume and potential energy we calculate $T_{g}$ as function of $Q_{r}$ for different, fixed pressures. We extrapolate our data to experimentally accessible quench rates and calculate the pressure dependence of the glass transition temperature. To check on the effect of the ensemble chosen for the simulation, we repeat some calcu- 


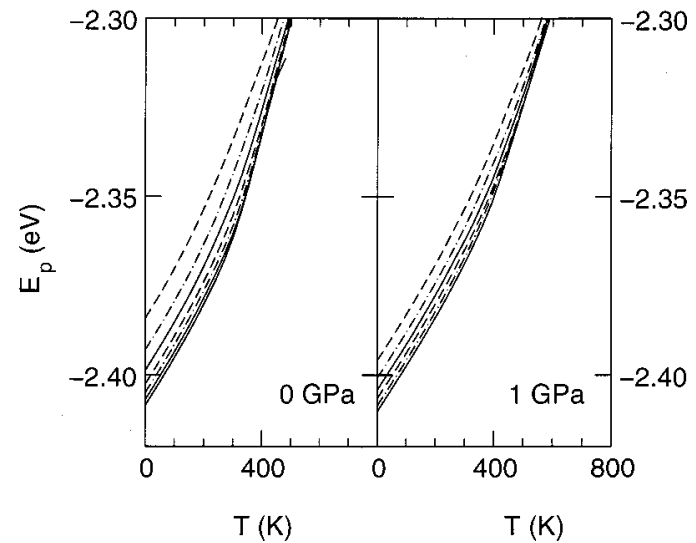

FIG. 1. Evolution of the potential energy per atom as function of temperature for different applied pressures $(p=0 \mathrm{GPa}$ left-hand side, $p=1 \mathrm{GPa}$ right-hand side) and different quench rates (from top to bottom: $Q_{r}=1$ $\times 10^{14}, 3 \times 10^{13}, 1 \times 10^{13}, 3 \times 10^{12}, 1 \times 10^{12}, 3 \times 10^{11} \mathrm{~K} / \mathrm{s}$ and $1 \times 10^{11} \mathrm{~K} / \mathrm{s}$ $(p=0$ only).

lations with fixed volume instead of fixed pressure. Finally, we summarize and conclude.

\section{CALCULATION}

We performed molecular dynamics simulations at fixed pressure (NPT ensemble) or fixed volume ( $N V T$ ensemble) of a system of 2000 atoms with periodic boundary conditions. The temperature is adjusted by velocity scaling and the pressure is fixed by the Parrinello-Rahman algorithm. ${ }^{15}$ To reproduce the interatomic interaction of Se we used a threebody potential previously introduced by Oligschleger et al. ${ }^{16}$ With this interaction one obtains amorphous structures of interlaced covalently bound chains and rings with a weak van-der-Waals-type interaction between them. ${ }^{10,11}$

The equations of motions are integrated with a Velocity Verlet algorithm. ${ }^{17}$ The time step is equal to $1 \mathrm{fs}$. More details about simulations can be found in Ref. 11.

We first equilibrated liquid samples at temperatures ranging from $T=650 \mathrm{~K}$ for $p=0$ to $T=1000 \mathrm{~K}$ for $p=6 \mathrm{GPa}$. The experimental melting temperature is at zero pressure $T_{m}=494 \mathrm{~K}$. During the quench we recorded the evolution of the potential energy and of the volume (simulations at fixed pressure) or pressure (simulations at fixed volume). Figure 1 shows the change of the potential energy per atom upon cooling for different quench rates and pressures of 0 and $1 \mathrm{GPa}$. As to be expected the potential energies per atom $\left(E_{p}\right)$ drop for slower quenches. The effect is reduced for the higher pressure. The total effect is rather small, which indicates that the quench rate does not strongly affect the number of covalent bonds between the Se atoms. Breaking a covalent bond would cost an energy of about $2.4 \mathrm{eV}$. Slowing the quench rate from $Q_{r}=3 \times 10^{11} \mathrm{~K} / \mathrm{s}$ to $Q_{r}=1 \times 10^{11} \mathrm{~K} / \mathrm{s}$ reduces the energy per atom only by $1 \mathrm{meV}$ at $p=0$ and less at higher pressures.

Increasing the quench rate the systems falls out of equilibrium at higher and higher temperatures. For the highest quench rate $\left(10^{14} \mathrm{~K} / \mathrm{s}\right)$ the system is not even in equilibrium at temperatures more than twice the glass transition temperature. (At such a fast quench rate details of the procedure used

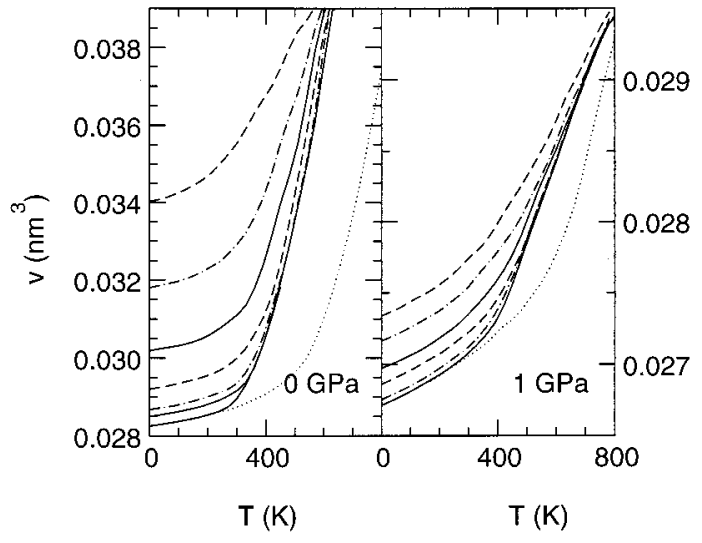

FIG. 2. Evolution of the specific volume per atom as function of temperature for different applied pressures $(p=0.0 \mathrm{GPa}$ left-hand side, $p=1 \mathrm{GPa}$ right-hand side) and different quench rates (from top to bottom: $Q_{r}=10^{14}$, $3 \times 10^{13}, 10^{13}, 3 \times 10^{12}, 10^{12}, 3 \times 10^{11} \mathrm{~K} / \mathrm{s}$, and $1 \times 10^{11} \mathrm{~K} / \mathrm{s}$ ( $p=0$ only). The dotted lines show the volume evolution upon rapidly heating the samples with the lowest quench rate.

to hold the pressure constant also become noticeable.) For the lowest quench rate $\left(3 \times 10^{11} \mathrm{~K} / \mathrm{s}\right) E_{p}(T)$ changes linearly with temperature both at low temperatures in the glass and at high temperatures in the liquid. The asymptotic slopes of the linear increase in the solid and in the liquid are independent of the quench rate; again an indication that the structure of the covalent bonding is not markedly changed. We did not check for the effects on the vibrational part. From our previous results ${ }^{10}$ we do not expect large effects. The transition region between the two linear slopes grows rapidly with quench rate. For the highest quench rate it stretches far into the hot liquid.

The corresponding plot for the evolution of the specific atomic volume $(v)$, Fig. 2, shows qualitatively the same behavior but the change of slope is more pronounced and the dependence on the quench rate is stronger, particularly for $p=0$. We observe a pronounced reduction of the atomic volume with applied pressure. The dotted line shows the evolution of the volume of the samples quenched with the lowest rates, when they are rapidly reheated (rate $10^{14} \mathrm{~K} / \mathrm{s}$ ), see the following.

\section{RESULTS}

\section{A. Constant pressure calculations (NPT ensemble)}

As in Ref. 18 we define the glass transition temperature, $T_{g}$, as the fictive temperature defined by the crossover of the extrapolations of the high and low temperature curves of energy or volume. Because of the larger effect we primarily use the temperature dependence of the volume. At the high quench rates of the simulations the crossover spans a large temperature interval which forces one, on the glassy side, to go beyond a linear extrapolation. In the previous work on LJG a quadratic fit was used. ${ }^{13}$ The volume expansion of amorphous Se is strongly nonlinear both in experiment ${ }^{19}$ and in our simulation. To account for this nonlinearity we calculate the temperature dependence of the volume, for each glassy sample and each quench rate, by rapidly heating from $T=0 \mathrm{~K}$ with a rate of $10^{14} \mathrm{~K} / \mathrm{s}$. The crossing of this curve 


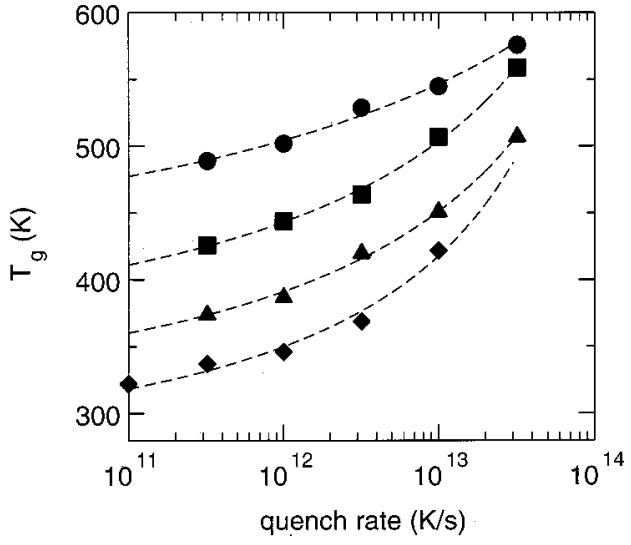

FIG. 3. Glass transition temperature, $T_{g}$, obtained from the evolution of volume (Fig. 2), as function of quench rate, $Q_{r}$, for different pressures (from bottom to top: $p=0,1,2$, and $4 \mathrm{GPa}$ ). The symbols depict the simulation values and the dashed line the fit by Eq. (1).

with the extrapolation from the liquid then defines $T_{g}$. This procedure is valid for quench rates much smaller than the heating rate, $Q_{r} \ll 10^{14} \mathrm{~K} / \mathrm{s}$, when the effects of configurational changes during heating are small compared to the ones occurring during the quench. This procedure separates the nonlinearity of the volume expansion inherent to the structure of the glass from the one due to relaxation. We neglect the small difference between "freezing temperature," $T_{Z}$, and $T_{g} \cdot{ }^{20}$

In Fig. 3 the resulting $T_{g}$ are plotted against $\log Q_{r}$ for different pressures. The expected increase with $Q_{r}$ is clearly visible. Reducing the quench rate from $Q_{r}=3 \times 10^{13} \mathrm{~K} / \mathrm{s}$ by an order of magnitude to $Q_{r}=3 \times 10^{12} \mathrm{~K} / \mathrm{s}$ reduces $T_{g}$ by $25 \%$. Slowing the quench rate by another order of magnitude, $T_{g}$ is reduced by less than $9 \%$.

To extrapolate to lower rates we use the procedure introduced by Vollmayr. ${ }^{3,13}$ Arguing that the glass transition occurs when the relaxation time increases upon cooling to the inverse of the quench rate, the dependence of $T_{g}$ on $Q_{r}$ can be fitted by a Vogel-Fulcher law

$$
T_{g}=T_{0}+\frac{B}{\ln A Q_{r}},
$$

where $A$ and $B$ are two parameters and $T_{0}$ is the glass transition temperature corresponding to $Q_{r} \rightarrow 0 \mathrm{~K} / \mathrm{s}$. The resulting fit is shown by the dashed lines in Fig. 3. The highest quench rates $\left(Q_{r}=10^{14} \mathrm{~K} / \mathrm{s}\right)$ were excluded from the fit. The fit gives estimates of $T_{0}=230,260,305$, and $350 \mathrm{~K}$ for the four pressures shown, respectively. We want to point out that these numbers are only rough guides. The fit is stabilized by the requirement of consistency between the values for different pressures. The values are, however, in a reasonable range and the value $T_{0}=230 \mathrm{~K}$ for $p=0$ is in agreement with the experimental one of $T_{0}=238 \mathrm{~K}^{21}$

The above-mentioned fit would predict for an experimental quench rate $Q_{r} \sim 1 \mathrm{~K} / \mathrm{s}$ glass transition temperatures $T_{g}=253,287,335$, and $394 \mathrm{~K}$. The value for $p=0$ is about $15 \%-20 \%$ lower than the experimental value.

Figure 4 shows the atomic volume at $T=0$ for different applied pressures as function of $T_{g}$. The linear dependence

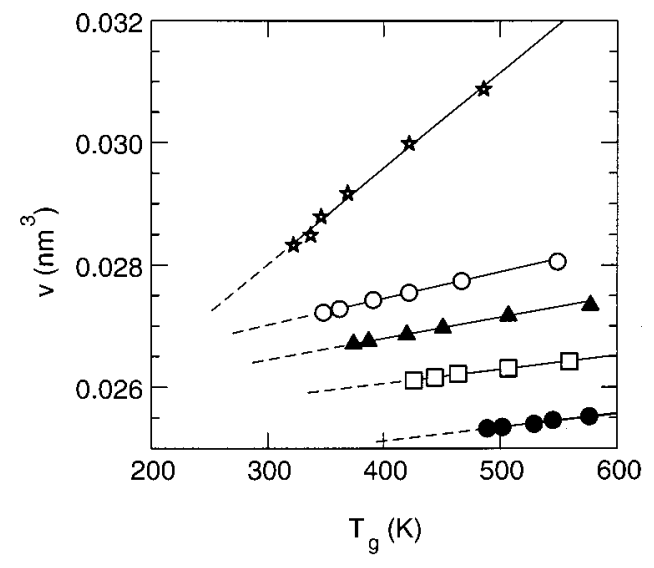

FIG. 4. Atomic volume at $T=0$ as function of $T_{g}$ for different pressures (from top to bottom: $0,0.5,1,2$, and $4 \mathrm{GPa}$ ). The symbols show the simulation results for $Q_{r}=10^{11}$ ( $p=0$ only), $3 \times 10^{11}, 10^{12}, 3 \times 10^{12}, 10^{13}, 3$ $\times 10^{13}$, and $10^{14} \mathrm{~K} / \mathrm{s}$. Solid lines: Least-squares fit to values up to $Q_{r}=3$ $\times 10^{13} \mathrm{~K} / \mathrm{s}$. Dashed lines: extrapolation to $T_{g}$ corresponding to $Q_{r}=1 \mathrm{~K} / \mathrm{s}$.

reflects the linearity of the expansion coefficients in the glass and the undercooled liquid. The slope of the lines is given by the difference of the two coefficients. The volume can be written as

$$
v\left(p, Q_{r}\right)=v_{0}(p)+\alpha(p) T_{g}\left(p, Q_{r}\right),
$$

with $T_{g}\left(p, Q_{r}\right)$ given by Eq. (1). With increasing pressure the slope of the $v\left(p, Q_{r}\right)$ lines rapidly decreases. In our previous work we have found that under zero pressure conditions may small voids are formed. They are seen as a small pre-peak in the structure factor. ${ }^{11}$ Diminishing the quench rate, the number of these voids decreases. The void formation can be suppressed by applying pressure. The smaller number of voids at higher pressures which can, therefore, be annealed by a slow quench, results in smaller slopes in Fig. 4. This effect saturates at pressures around $2 \mathrm{GPa}$, and the slope no longer diminishes. Also the pre-peak in the structure factor vanishes. ${ }^{11}$

The linear dependence in Fig. 4 allows us to extrapolate to low quench rates (dashed lines). For $Q_{r}=1 \mathrm{~K} / \mathrm{s}$ and $p=0$ we thus find an atomic volume of $v=0.027 \mathrm{~nm}^{3}$ which is about $10 \%$ too low, compared to experiment. ${ }^{19}$ This suggests that one should scale the energy and length parameters in the interatomic potential by $10 \%$ and $3 \%$, respectively. Such a change would not affect the elastic properties (dimension energy/length ${ }^{3}$ ) and only weakly the vibrational frequencies $\left(\nu^{2} \propto\right.$ energy/length $\left.{ }^{2}\right)$. It would be compatible with the fit of the molecular binding energies used in the construction of the potential. ${ }^{16}$ Another possibility is that the fitted values of $T_{0}$ are too low. An increase by $10 \%$ would still be within the fitting accuracy.

Repeating the determination of $T_{g}$ using the potential energy, Fig. 1, we get the same picture. At the higher quench rates there is a tendency for $T_{g}$, calculated this way, to be slightly lower than the previous values obtained from the evolution of volume with temperature. The difference between $T_{g}$ calculated from the evolution of the volume or the energy drops below the accuracy of our calculation around 


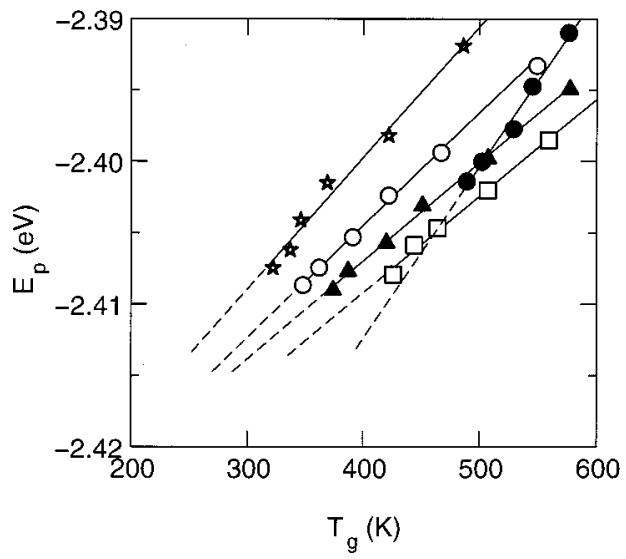

FIG. 5. Atomic enthalpy at $T=0$ as function of $T_{g}$ for different pressures (from top to bottom: $0,0.5,1,2$, and $4 \mathrm{GPa}$ ). The symbols show the simulation results for $Q_{r}=10^{11}$ ( $p=0$ only), $3 \times 10^{11}, 10^{12}, 3 \times 10^{12}, 10^{13}, 3$ $\times 10^{13}$, and $10^{14} \mathrm{~K} / \mathrm{s}$. Solid lines: least-squares fit to values up to $Q_{r}=3$ $\times 10^{13} \mathrm{~K} / \mathrm{s}$. Dashed lines: extrapolation to $T_{g}$ corresponding to $Q_{r}=1 \mathrm{~K} / \mathrm{s}$.

$Q_{r}=10^{11} \mathrm{~K} / \mathrm{s}$. This behavior is reminiscent of results in polymers where different activation energies for volume and shear deformations have been observed. ${ }^{22}$

The potential energy per atom at $T=0$, Fig. 5 , shows the same linear behavior as observed for the volume in Fig. 4. Therefore the energy can be written analogously as

$$
E_{p}\left(p, Q_{r}\right)=E_{0}(p)+\alpha_{E}(p) T_{g}\left(p, Q_{r}\right) .
$$

Raising the pressure we find first a weak decrease of slope, then a saturation between 1 and $2 \mathrm{GPa}$, and finally a strong increase. This can be understood from the different energy scales governing the behavior of materials such as Se. As we have discussed in connection with Fig. 4, at low pressures slower quenches mean fewer voids. This in turn will increase the attraction between the Se rings and chains. At higher pressure this effect diminishes and weak repulsive interchain forces come into play. At the highest pressures, finally, the strong intrachain interactions will be stressed. This is reflected in the rapid increase of the slope for $p \rightarrow 4 \mathrm{GPa}$. From Fig. 5 one can derive for a given quench rate the pressure which will produce the "best" (lowest energy) glass at $T=0$. This "optimal" pressure would be between 0.5 and $1 \mathrm{GPa}$. An optimal unpressurized glass can be obtained by slowly releasing the pressure at some given temperature, well below $T_{g}$. If we do this at $T=0$ for our samples, produced with $Q_{r}=3 \times 10^{11} \mathrm{~K} / \mathrm{s}$, we find the lowest energy for the samples quenched under a pressure of $2 \mathrm{GPa}$. We expect that this pressure would be reduced if the pressure were released at higher temperatures.

Once we have the value of $T_{g}$ at a given pressure for a given $Q_{r}$, we are able to draw the line of transition between the liquid and the glass, the closed symbols in Fig. 6. Additionally shown is $T_{g}$ extrapolated to $Q_{r}=1 \mathrm{~K} / \mathrm{s}$. The pressure dependence of $T_{g}$ is not strongly affected by the quench rate. An increase in pressure of $1 \mathrm{GPa}$ raises $T_{g}$ by nearly $15 \%$.

\section{B. Constant volume calculations (NVT ensemble)}

For ease of calculation, simulations are frequently done with fixed volume instead of constant pressure. To check on

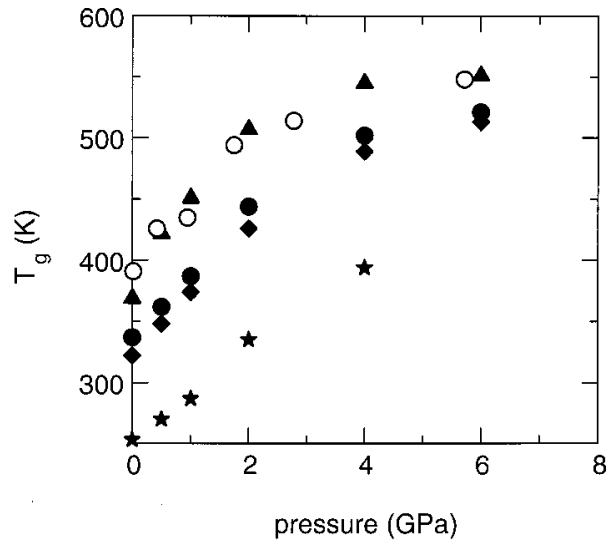

FIG. 6. $T_{g}$ as a function of the pressure. Closed symbols: quench under constant pressure with quench rates $3 \times 10^{11}$ (diamonds), $10^{12}$ (circles), and $10^{13} \mathrm{~K} / \mathrm{s}$ (triangle). Open circle: quench with constant volume condition plotted against pressure at $T=0, Q_{r}=10^{12} \mathrm{~K} / \mathrm{s}$. The stars denote the values obtained from the extrapolation to $Q_{r}=1 \mathrm{~K} / \mathrm{s}$.

the effect of the chosen ensemble we repeated some calculations using the $N V T$ ensemble. Instead of defining the glass transition temperature from the change of volume expansion we used the change of the pressure derivative (compressibility) as an indication of structural arrest. The resulting $T_{g}$ for $Q_{r}=10^{12} \mathrm{~K} / \mathrm{s}$ are shown by the open circles in Fig. 6 . As is common practice, we plot $T_{g}$ against the pressure at $T=0$. The pressure dependence in the $N V T$ ensemble $\left(Q_{r}=10^{12} \mathrm{~K} / \mathrm{s}\right)$ is similar to the one of the NPT ensemble. The magnitude of the $T_{g}$ values corresponds, however, to a ten times faster quench rate in the $N P T$ ensemble. If one would plot against the pressure at the $T_{g}$ this upward shift would more or less disappear. This can easily be understood taking into account that the structure is frozen-in at $T_{g}$. This point of view is confirmed when one compares the atomic volumes and potential energies at $T=0$. For $p=0$ in the $N V T$ ensemble we get $V_{\text {at }}=0.027 \mathrm{~nm}^{3}$ and $E_{p}=-2.411 \mathrm{eV}$. The $N P T$ ensemble gives for $p=0$ the values $V_{\text {at }}=0.028 \mathrm{~nm}^{3}$ and $E_{p}=-2.406 \mathrm{eV}$ and for $p=1 \mathrm{GPa}$ the values $V_{\text {at }}=0.027 \mathrm{~nm}^{3}$ and $E_{p}=-2.408 \mathrm{eV}$. Clearly the zero pressure $N V T$ ensemble corresponds to a $N P T$ ensemble at high pressure, $p=1 \mathrm{GPa}$ or slightly above.

A way out would be to take the pressure at $T_{g}$ to characterize the $N V T$ simulation. It is, however, not sensible to fix the volume in an $N V T$ ensemble to $P\left(T_{g}\right)=0$ since this implies a negative pressure in the glassy state. In a loosely bounded material such as Se this leads to a breakdown of the structure as already indicated by the strong increase in volume for $p \rightarrow 0$, Fig. 2 .

\section{DISCUSSION}

Extrapolated to experimentally accessible quench rates, we find a good overall agreement of our simulation with experiment. In particular, the glass transition temperature agrees within $20 \%$.

There are not many experiments on the effect of cooling rate on the glass transition. Changing $Q_{r}$ from 0.5 to 0.005 $\mathrm{K} / \mathrm{min}$, Dzhalilov and Rzaev ${ }^{23}$ report a shift of $T_{g}$ by $9 \mathrm{~K}$ (about 2.9\%) whereas we find a shift of only $0.8 \%$. This 
difference by a factor 3 is outside the possible error margin applying Eq. (1). The difference might be partially due to the different definitions of $T_{g}$. Dzhalilov and Rzaev use extrapolations over a range of $40 \mathrm{~K}$ around $T_{g}$ whereas we use extrapolations from $T=0$ and high temperatures, respectively. On the glassy side the slope of the extrapolation lines is nearly independent of the quench rate in our simulation. Dzhalilov and Rzaev find, in the small interval of about 20 $\mathrm{K}$, a marked change of slope.

Brüning et al. ${ }^{24}$ investigated the change of enthalpy upon aging a glass for $14 \mathrm{~h}$ at $T=300 \mathrm{~K}$ and find an increase in density by $0.14 \%$ and a lowering of enthalpy by $1 \mathrm{meV} /$ atom. We would expect to find similar values upon changing the quench rate from the experimental value of $10 \mathrm{~K} / \mathrm{min}$ to something like $1 \mathrm{~K} / \mathrm{h}$. Doing this we find for our simulation a change of atomic volume of $0.17 \%$ and a change of enthalpy of $0.4 \mathrm{meV}$.

From experiment an increase by $13 \mathrm{~K}$ for a pressure of $200 \mathrm{MPa}$ was reported. ${ }^{25}$ This would correspond to a $20 \%$ increase for $1 \mathrm{GPa}$. Increasing the pressure from 0 to $1 \mathrm{GPa}$ we find an increase of $T_{g}$ by $13.5 \%$ in fair agreement with experiment.

In simple soft sphere systems $\left[V(R)=\epsilon(\sigma / R)^{12}\right]$ a scaling of the glass transition temperature with $1 /\left(v T^{1 / 4}\right)$ has been observed both in simulations ${ }^{26}$ and in experiment. ${ }^{27}$ In a one atomic Lennard-Jones system the diffusional isotope effect, which measures the collectivity of the motion, was found to scale with density. ${ }^{28}$ In a binary system at low pressures such a scaling no longer holds, the scaling for the two components differs strongly. ${ }^{29}$ For Se at low pressures, density and energy are decoupled, as seen comparing Figs. 1 and 2. This is a consequence of the chain structure which allows the creation of voids with a near zero formation energy. Therefore, one cannot expect simple scaling with density.

\section{CONCLUSION}

To conclude we have shown that for an NPT ensemble the molecular dynamics simulation for the given model of Se predicts the glass transition temperature and the specific volume (density) within 20\% accuracy. In agreement with experiment, we observe a strong increase of $T_{g}$ with applied external pressure.

At fixed pressure, the glass transition temperature decreases with $Q_{r}$ as expected from experiment. This decrease leads to a reasonable value of $T_{g}$ when $Q_{r}$ tends to 0 . We have drawn the transition line between the liquid and the glassy phases. Working in NPT or NVT ensemble is not equivalent. Working under fixed volume conditions ( $N V T$ ensemble) we get glass transition temperatures in Se which correspond to quench rates which are an order of magnitude faster than in the NPT ensemble. This effect will be general, its magnitude should be checked for different materials.

The specific volume and enthalpy depend linearly on the quench-rate-dependent glass transition temperature. The pressure dependence of the slopes reflects the tendency to void formation and the different magnitudes of inter- and intrachain interactions.

\section{ACKNOWLEDGMENT}

One of us (D.C.) would like to thank the A. von Humboldt foundation for its support.

${ }^{1}$ B. B. Laird and H. R. Schober, Phys. Rev. Lett. 66, 636 (1991); H. R. Schober and B. B. Laird, Phys. Rev. B 44, 6746 (1991); H. R. Schober and C. Oligschleger, ibid. 53, 11469 (1996); P. Jund, D. Caprion, and R. Jullien, Europhys. Lett. 37, 547 (1997); D. Caprion, P. Jund, J.-P. Bonnet, and R. Jullien, J. Non-Cryst. Solids 232-234, 459 (1998); C. Oligschleger and H. R. Schober, Phys. Rev. B 59, 811 (1999).

${ }^{2}$ W. Kob and H. C. Andersen, Phys. Rev. Lett. 73, 1376 (1994); W. Kob and H. C. Andersen, Phys. Rev. E 51, 4626 (1995); 52, 4134 (1995); V. Mazzacurati, G. Ruocco, and M. Sampoli, Europhys. Lett. 34, 681 (1996); W. Kob and J.-L. Barrat, Phys. Rev. Lett. 78, 4581 (1997); S. Sastry, P. G. Debenedetti, and F. H. Stillinger, Nature (London) 393, 554 (1998); C. Donati, J. F. Douglas, W. Kob, S. J. Plimpton, P. H. Poole, and S. C. Glotzer, Phys. Rev. Lett. 80, 2338 (1998); J.-L. Barrat and W. Kob, Europhys. Lett. 46, 637 (1999).

${ }^{3}$ K. Vollmayr, W. Kob, and K. Binder, Europhys. Lett. 32, 715 (1995).

${ }^{4}$ W. Jin, P. Vashishta, R. K. Kalia, and J. P. Rino, Phys. Rev. B 48, 9359 (1993); J. Horbach, W. Kob, and K. Binder, Philos. Mag. B 77, 297 (1998); S. N. Taraskin and S. R. Elliott, Phys. Rev. B 59, 8572 (1999); P. Jund and R. Jullien, ibid. 59, 13707 (1999); Phys. Rev. Lett. 83, 2210 (1999), C. Oligschleger, Phys. Rev. B 60, 3182 (1999).

${ }^{5}$ K. Vollmayr, W. Kob, and K. Binder, Phys. Rev. B 54, 15808 (1996).

${ }^{6}$ J. Horbach and W. Kob, Phys. Rev. B 60, 3169 (1999).

${ }^{7}$ H. Teichler, Phys. Rev. Lett. 76, 62 (1996).

${ }^{8}$ C. Gaukel and H. R. Schober, Solid State Commun. 107, 1 (1998); C. Gaukel, M. Kluge, and H. R. Schober, J. Non-Cryst. Solids 250-252, 664 (1999).

${ }^{9}$ P. Vashishta, R. K. Kalia, and I. Ebbsjo, Phys. Rev. B 39, 6034 (1989); P. Vashista, R. K. Kalia, G. A. Antonio, and I. Ebbsjo, Phys. Rev. Lett. 62, 1651 (1989).

${ }^{10}$ N. G. Almarza, E. Enciso, and F. J. Bermejo, Europhys. Lett. 17, 595 (1992); C. Oligschleger and H. R. Schober, Physica A 201, 391 (1993); C. Oligschleger and H. R. Schober, Solid State Commun. 93, 1031 (1995); H. R. Schober, C. Gaukel, and C. Oligschleger, Prog. Theor. Phys. Suppl. 126, 67 (1997); C. Oligschleger and J. C. Schön, J. Phys.: Condens. Matter 9, 1049 (1997); T. Koslowski, Z. Phys. Chem. (Munich) 210, 45 (1999).

${ }^{11}$ D. Caprion and H. R. Schober, Phys. Rev. B 62, 3709 (2000).

${ }^{12}$ S. Mossa, R. Di Leonardo, G. Ruocco, and M. Sampoli, Phys. Rev. E 62, 612 (2000).

${ }^{13}$ K. Vollmayr-Lee, W. Kob, K. Binder, and A. Zippelius, Int. J. Mod. Phys. C 10, 1443 (1999).

${ }^{14}$ U. Buchenau and R. Zorn, Europhys. Lett. 18, 523 (1992).

${ }^{15}$ M. Parrinello and A. Rahman, Phys. Rev. Lett. 45, 1196 (1980); M. Parrinello and A. Rahman, J. Appl. Phys. 52, 7182 (1981).

${ }^{16}$ C. Oligschleger, R. O. Jones, S. M. Reimann, and H. R. Schober, Phys. Rev. B 53, 6165 (1996); note that parameter $a$ in Table I should read 9281.2.

${ }^{17}$ W. C. Swope, H. C. Andersen, P. Berens, and K. R. Wilson, J. Chem. Phys. 76, 637 (1982).

${ }^{18}$ G. W. Scherer, in Materials Science and Technology, edited by R. W. Cahn, P. Haasen and E. J. Kramer, Glasses and Amorphous Materials Vol. 9, edited by J. Zarzycki (VCH, Weinheim, 1991), p. 119.

${ }^{19}$ R. Grosse, P. Krause, M. Meissner, and A. Tausend, J. Phys. C 11, 45 (1978).

${ }^{20}$ A. Feltz, Amorphous Inorganic Materials and Glasses (VCH, Weinheim, 1993).

${ }^{21}$ Kh. M. Khalikov and B. B. Kuliev, Fiz. Tverd. Tela (Leningrad) 7, 2487 (1965).

${ }^{22}$ R. Kono, J. Phys. Soc. Jpn. 15, 718 (1960); 16, 1580 (1961).

${ }^{23}$ S. U. Dzhalilov and K. I. Rzaev, Phys. Status Solidi 20, 261 (1967).

${ }^{24}$ R. Brüning, E. Irving, and G. LeBlanc, J. Appl. Phys. 89, 3215 (2001).

${ }^{25}$ J. I. Berg and R. Simha, J. Non-Cryst. Solids 22, 1 (1976).

${ }^{26}$ B. Bernu, J.-P. Hansen, Y. Hiwatari, and G. Pastore, Phys. Rev. A 36, 4891 (1987).

${ }^{27}$ A. Tölle, H. Schober, J. Wuttke, O. G. Randl, and F. Fujara, Phys. Rev. Lett. 80, 2374 (1998).

${ }^{28}$ M. Kluge and H. R. Schober, Phys. Rev. E 62, 597 (2000).

${ }^{29}$ H. R. Schober, Solid State Commun. 119, 73 (2001). 